\title{
Wearable sensor-based data analysis for neurological disease symptoms evaluation utilising quantitative approach.
}

\author{
Mariusz Chmielewski ${ }^{1, \mathrm{a}}$, Michat Nowotarski ${ }^{1, \mathrm{~b}}$ \\ ${ }^{1}$ Military University of Technology, Cybernetics Faculty, gen. W. Urbanowicz Street 2, Warsaw, Poland
}

\begin{abstract}
The paper describes implementation of an analytical method and conclusions of novel approach to clinical trials monitoring and evaluation. Based on clinical trials observations a set of requirements for validating symptoms of neurological diseases have been formulated, concentrating on the ones which can be registered using wearable sensors. The constructed tool utilizes conventional surveying methods supplemented with biomedical sensor for neurological symptoms recognition and intensity evaluation. Developed mobile system is aimed at clinical trials assistance utilising sensor-based state evaluation. Such quantitative approach is a supplement for patient's subjective evaluation of health state. This work is a discussion on pros and cons of such process composition and its supplementation with technology. Existing methodology relies on health state evaluation based on iteratively answered questionnaires, which in our understanding cannot be fully controlled and reliable. Utilisation of actigraphy and electromyography provides efficient means of some gestures recognition but most of all PD tremor identification and evaluation of their intensity, therefore can be used for ON/OFF state and dyskinesia identification and evaluation. In order to recognise specific states for PD patients (tremors, bradykinesias, rigidity, mental slowness, etc.) a set of additional techniques have been designed and implemented.
\end{abstract}

\section{Introduction and research motivation}

The paper contains description of research assumptions, construction details and obtained experiences during the conceptual and testing phase of a PATRON [8], [1], [17] system, which is aimed at acquisition, identification, recognition and assessment of specified neurological disease symptoms. Implemented method concentrates on detecting Parkinson's Disease tremors, arm rigidness and some forms of bradykinesias [20]. This is achieved by application of wearable biomedical sensors monitoring inertia and muscle activity and tonicity. Several research papers have been published in that area [2], [3], [1] with respect to their original analytical methodology. The most important, original findings is this work provide three correlating aspects of pharmaceutical therapy evaluation:

1. Patient subjective health state evaluation - based on the patient survey responses,

2. Tremor recognition and intensity evaluation based on the inertial and biomedical sensor application and processing

3. Reflex and mental perception assessment - based on the touch screen exercise execution and efficiency testing, providing complemental assessment of patient.
The tool provides mechanisms to schedule all three aspects correlating prescribed therapy (mono or poly therapy) assigned to patient in conducted clinical trial. PATRON can also be used by medical institutions to assess efficiency of treatment providing alternative usage scenario.

The scheduling mechanisms determine how many times in an hour, a given request for data/evaluation is generated - notifying the user to perform actions on survey, sensor examination or perception test. To increase the usability of the tool a postpone option has been provided, which allows the patient to reschedule the action while recording that event in application logs. All user actions depend on accurate scheduling and interactions, thus the application record every notification, patient reaction and recorded data - providing detailed auditing. By default patient is required to answer 2-4 surveys per hour, execute sensoraugmented survey once an hour and evaluate his perception every two hours. Notifications about the drug intake and meals are tuned by the therapy (clinical trial) coordinator and can be adjusted based on the daily reports and $\mathrm{ON} / \mathrm{OFF}$ state correspondences [20]. The main requirement for PD therapy monitoring is continuous handheld and wearable assistance thus the sensors and mobile device have been configured good readability (supported with voice guidance) (Fig.2, Fig.3, Fig.4) and

\footnotetext{
${ }^{a}$ Mariusz Chmielewski: mchmielewski@wat.edu.pl

b Michał Nowotarski: michal.nowotarski@,wat.edu.pl
} 
seamless usage. Implemented method requires from user/patient strict compliance with the surveys and examinations, hence the notification subsystem, application support for action assistance and detailed logging. All audited events, interactions are locally stored for later inspection and application self-diagnostics. For analysis the application exports all events (and collected sensor-data sets in form of CSV files - examination metadata and scheduled events).

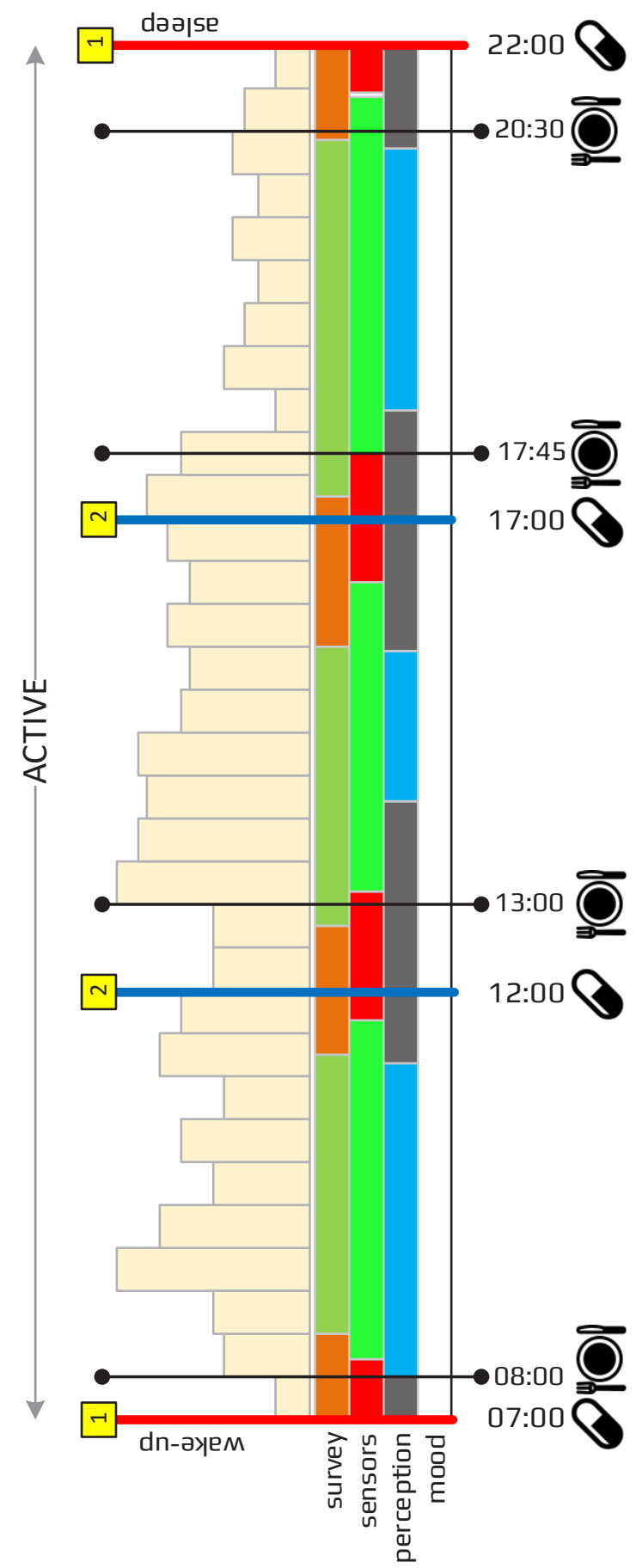

Fig. 1. A daily aggregated view of patient health state and registered events - from left - actigraphy histogram, survey, sensor, perception test - evaluations and optional mood data correlated with drug intake and meals.

\section{Sensor-based symptoms evaluation}

The initial development of the system was based on registering and monitoring the patient's continuous condition on the basis of sensors from a smartphone and smartwatch. Main sensors were accelerometer and gyroscope, which are currently placed in most of today's smartphones and smartwatches. It provided possibility to record information about the movement of patient's hand on his request. During the development, the system was expanded with the Myo armband [9]. Along the accelerometer's data of wrist/forearm movement an eight channel surface electromyography.

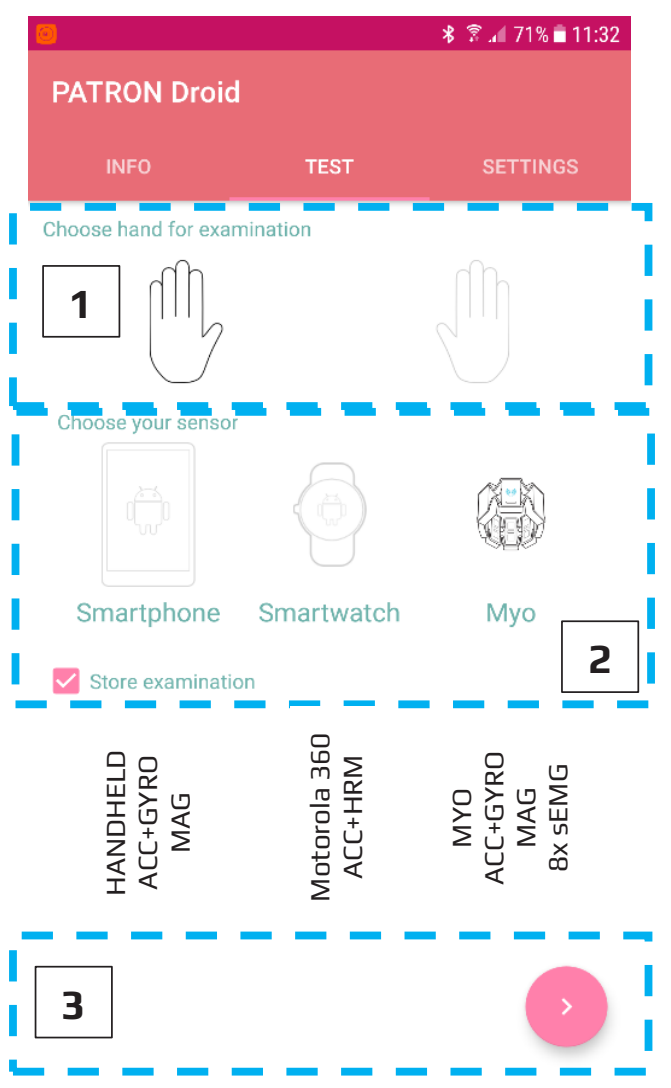

Fig. 2. View of examination preliminary phase - the sensorbased examination requires definition of measurements location (1), sensor data sources (2) (smartphone, smartwatch, MYO sensor), examination control (3).

Based on gathered test data it was determined that using the armband, it is possible to make a simple diagnosis of symptoms of selected neurological diseases. It was based on a simple mechanism analysing received signals and searching for the occurrence of certain health related events. If the event occurred during test, the appropriate message related to the registered signal was displayed for the user. The mechanism was based on the following signal features [2][3]:

minimum - maximum values, average and std. dev. from signal module, number of samples which exceeded threshold (adaptive) values. 


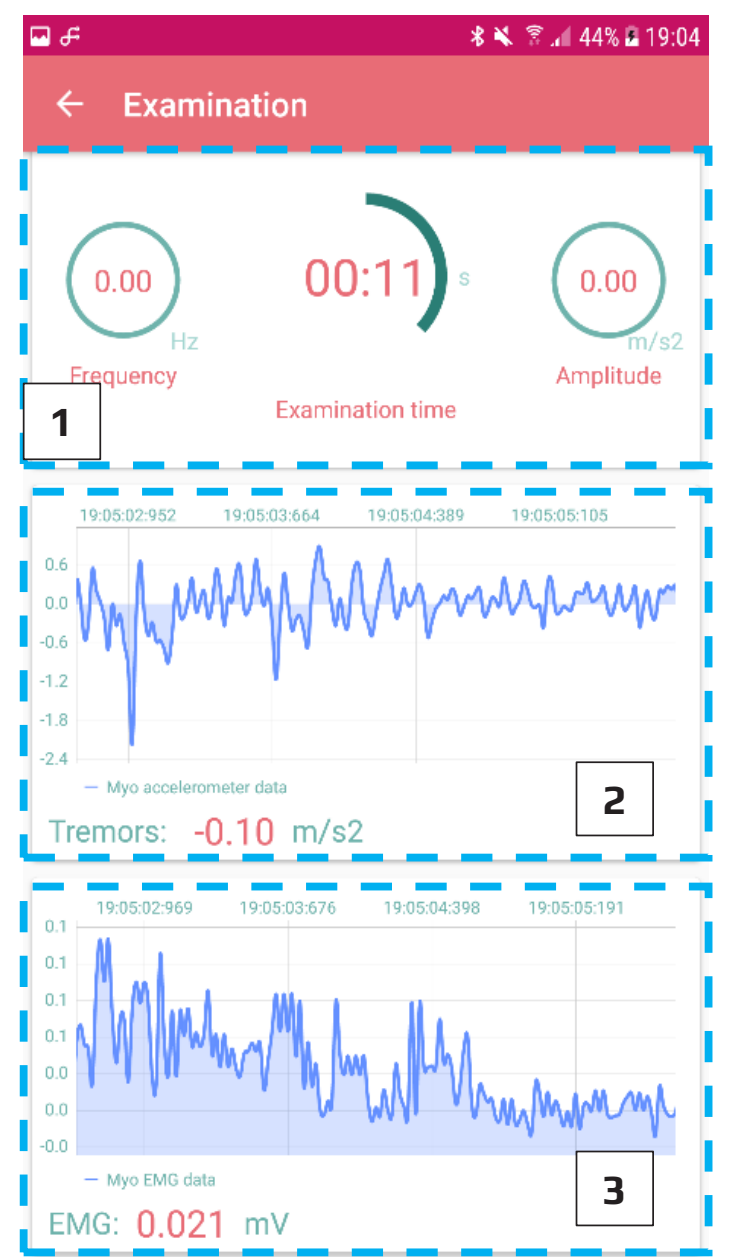

Fig. 3. Sensor assisted examination procedure -1 examination status with aggregated evaluation measures, 2 - inertial signal $|\mathrm{v}|$ for actigraphy assessment 3 -8-channel sEMG module signal

During method's test phase, above set of features has been extended as it was not sufficient enough (only $37 \%$ of accuracy) to provide adequate diagnose. A significant part of the relevant data was lost and obtained results had a significant measurement error. Further search for useful signal characteristics was performed [13],[14], resulting in selecting more complex and signal specific features for ACC and EMG data [4],[5]: number of zero crossings, mean waveform length, mean absolute, maximum amplitude, median frequency, mean frequency, peak frequency, signal power.

A comparison of the effectiveness of utilised previously selected set of signal features has been presented in [1] and [17]. The evaluation process evaluated for similar test cases. The second group of characteristics allow algorithms for more detailed analysis of recorded inertial and biomedical signals, which is a crucial characteristics in terms of evaluating the intensity of specific disease symptoms. It should be also stated that selected measuring techniques concentrate on measuring the effects of muscles activity, as a result of degrading extrapyramidal motor system [20].

In-depth research on effectiveness and usefulness of selected characteristics and monitored symptoms of PD patients have been described in [1], [17] and [3], [4]. This research however goes further, as it introduces monitoring of intentionally instructed exercises (arm vertical and horizontal movements, etc. performed to identify rigidness) as well as reflex testing using screen-based interactions (Fig. 4).

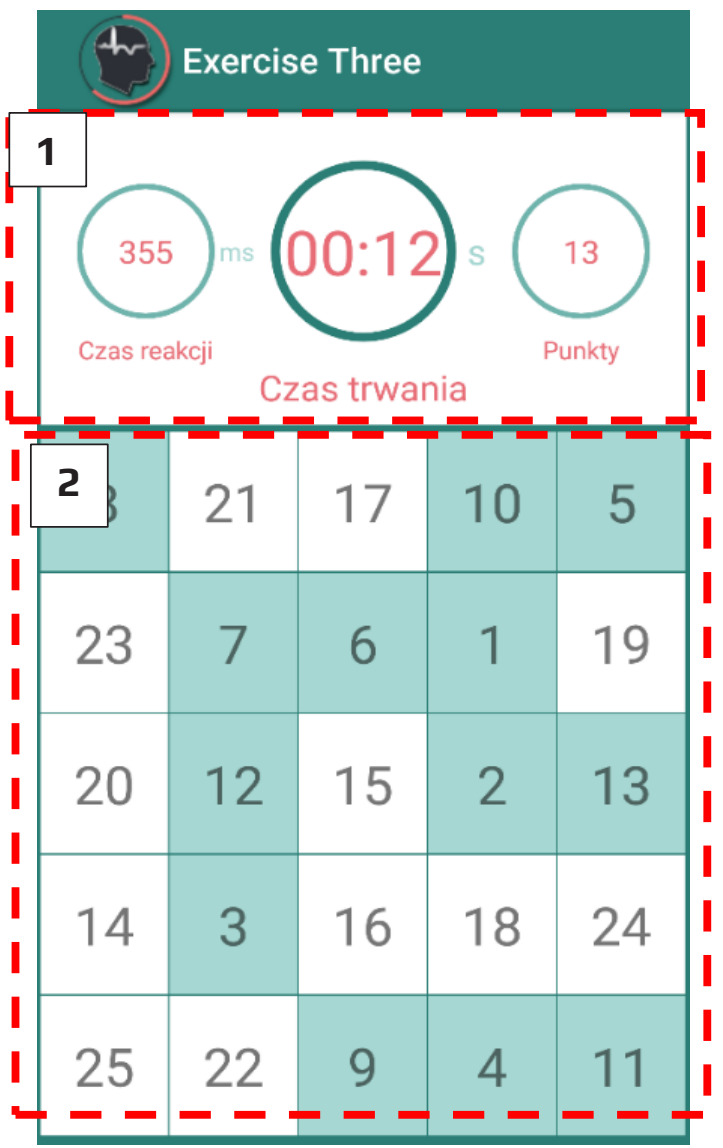

Fig. 4. Reflex and mental perception test (1 of 5 exercises). Randomised table of numbers requires to effectively select (touch) numbers in ascending order. In other variants numbers are being rendered in various screen regions.

\section{Software mechanisms for data acquisition and disease symptoms evaluation}

By default the examination is started with a configurable delay to set up connection between smartphone and MYO sensor. During that period the mobile device is configuring the communication channel and data source which will be recorded. Each device consist of several sensors (both handheld and wearables).

Depending on stored in application configuration specified sensors are recorded based on event notifications from SensorManager or based on queries sent through BLE channel.

Described application is dedicated for Android OS with API $>21$ and carries out tests based on both inertial and sEMG signals thus requires rich sensor support from the handheld device itself. The application utilizes BLE communication channel for acquiring measurements form multi-sensor wearable devices. 
The application delivers two approaches to record the patient's condition. The first one, subjective, based on the iterative, cyclic diagnostic survey (4 questions) completed by the patient and objective (quantitative), using examination procedures and sensor-based data described further in the paper.

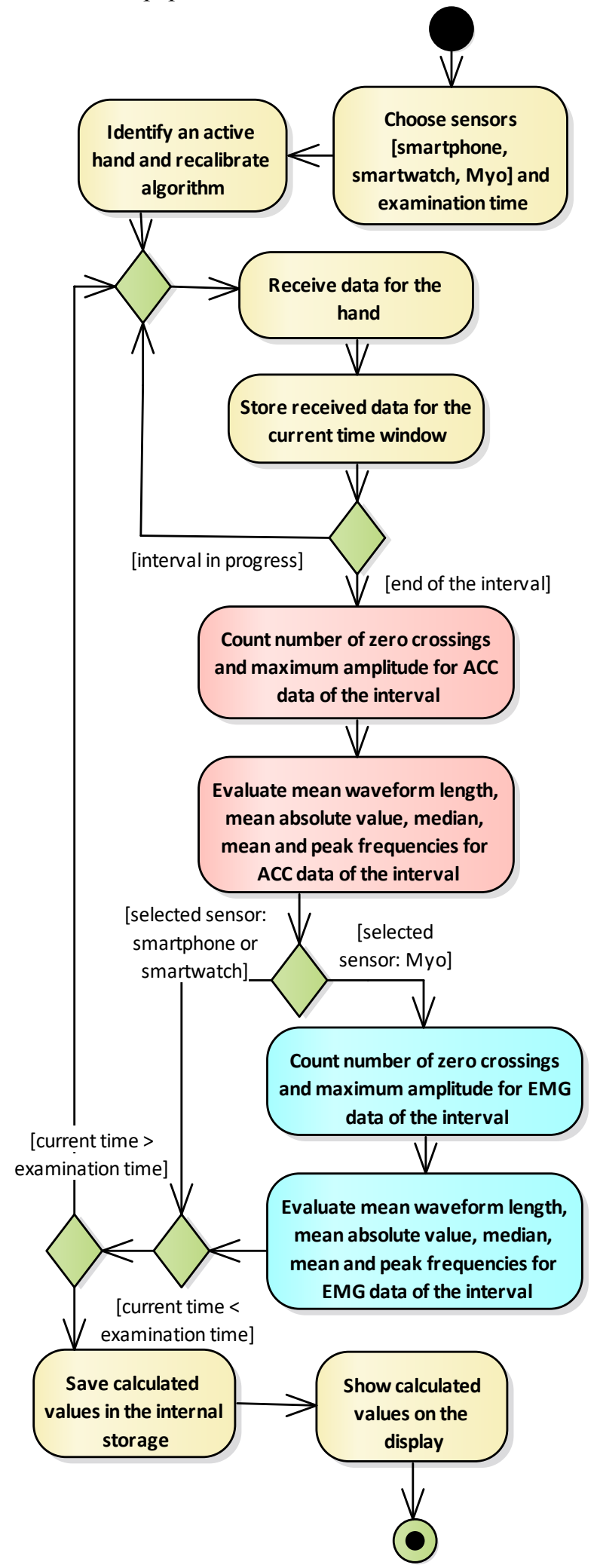

Fig. 5. Sensor-augmented examination algorithm
Before performing the test, the patient is asked to perform initial configuration of the application and calibration of threshold sensor values for ON/OFF and dyskinesias. The calibration process is optional, but ensures accuracy of recognition algorithms to patient specific needs and symptoms. It is necessary to pair devices with an app, calibrate sensors and set the test configuration.

During the test, the visualization of the received data is presented. On the user's request, it is possible to save received signals to a file stored on the smartphone. When the test is being completed, a signal analysis is performed to determine the characteristics described before. Calculated values are stored as an csv file in the smartphone's external storage.

\section{Research methodology}

Based on previous experiences, the research methodology has been modified. Concentration on Instead of the earlier approach, which aimed at the diagnosis of the symptoms of selected diseases (Parkinson's disease and epilepsy), the research is directed at detecting the occurrence and differentiation of certain patterns. In addition, instead of analysing aggregated accelerometer and electromyograph signals, individual axes for the accelerometer and each of the eight channel sEMG have been analysed separately. This approach enables a more flexible verification of the used classifiers and allows researchers to obtain accurate analysis of the patient's hand behaviour. Identification of PD tremors and bradykinesias rely on specific hand movements which in majority cases are simple thus the method itself should rely on more complex hand gestures (movements) closely associated with test exercises or rhythmic, muscle activity involving oscillations or twitching movements.

Several test behaviours have been selected and elaborated during the tests. The 9- The aim of the research was to check whether the applied classifiers would allow the tester to detect the execution of the intended event and identify it.

- Test \#1: Hand held steady and still

- Test \#2: A gentle hand shake

- Test \#3: Fist clenched with all strength

- Test \#4: Waving an open hand

- Test \#5: Deflection of hand to back

- Test \#6: Waving a clenched fist

Based on these test scenarios it was possible to verify the classification capability of the method but most of all the sensitivity of disease states recognition process. For preliminary recognition phase characteristics [1],[17] set of algorithms have been provided.

\section{Evaluation scenarios observations}

Tests (sensor-fused examinations) presented in the current paragraph were carried out for the test cases listed in the paragraph 4. 


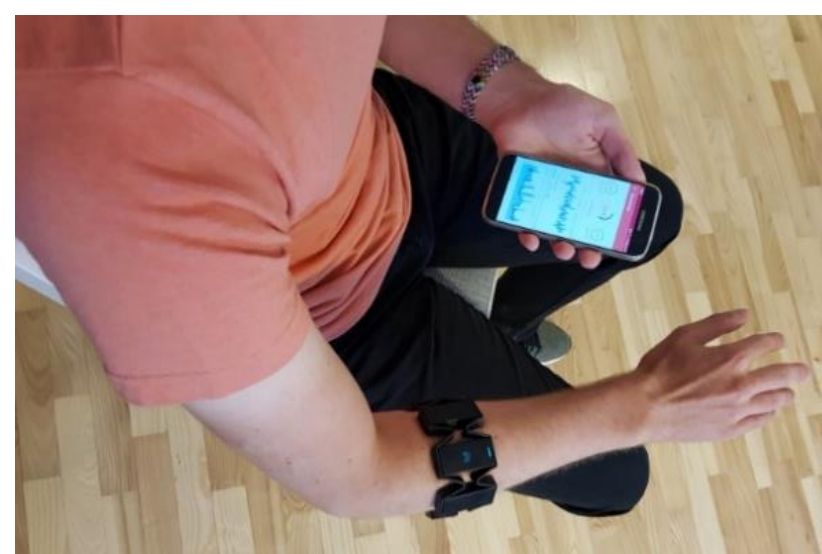

Fig. 6. PATRON collecting data with MYO sensor during the diagnostic-monitoring phase (both devices can be used for data acquisition - for asymmetric behaviour identification)

All tests were conducted for 2 minute duration, while signal features were calculated in 20 -second intervals. For each test case (Fig.3), results for one the most representative interval are presented. Tests were performed by the paper authors: males, 30-40 years old, physically active with low body fat and without any neurological, muscle disabilities. Examinations were repeated for each tester 15 times with 5 min delays to exclude muscle system fatigue. Similar procedures have been applied for reflex evaluations (Fig.4), to supplement gathered data with, a diagnostic factor describing the effects of long term, multiple, tedious sensor-fused examinations.

\subsection{Evaluation Test Scenario \#1}

The test was to record the signals of the person holding the hand horizontally and motionless.

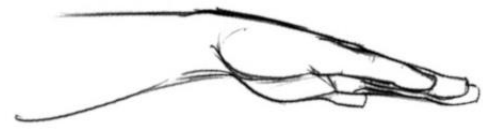

Fig. 7. Test gesture \#1 - Hand held steady and still with variant showing fatigue tremor

Table 1. ACC-sEMG signal features gathered for test gesture \#1 (avg values).

\begin{tabular}{|c|c|c|c|c|}
\hline \multicolumn{5}{|c|}{ Accelerometer } \\
\hline \multicolumn{2}{|l|}{ Axis } & $\mathbf{X}$ & $\mathbf{Y}$ & $\mathbf{Z}$ \\
\hline \multicolumn{2}{|l|}{ No. of zero crossings } & 254 & 295 & 242 \\
\hline \multicolumn{2}{|l|}{ Mean waveform length [s] } & 0.07 & 0.06 & 0.07 \\
\hline \multicolumn{2}{|l|}{ Mean absolute value $\left[\mathrm{m} / \mathrm{s}^{2}\right]$} & 0.02 & 0.02 & 0.03 \\
\hline \multicolumn{2}{|l|}{ Maximum amplitude $\left[\mathrm{m} / \mathrm{s}^{2}\right]$} & 0.08 & 0.10 & 0.10 \\
\hline \multicolumn{2}{|l|}{ Median frequency [Hz] } & 23.26 & 24.39 & 23.81 \\
\hline \multicolumn{2}{|l|}{ Mean frequency [Hz] } & 18.91 & 23.17 & 20.71 \\
\hline \multicolumn{2}{|l|}{ Peak frequency [Hz] } & 0.00 & 0.00 & 0.00 \\
\hline \multicolumn{5}{|c|}{ Electromyograph } \\
\hline Electrode & 1 & 2 & 3 & 4 \\
\hline No. of zero crossings & 145 & 138 & 155 & 145 \\
\hline Mean waveform length [s] & 0.12 & 0.13 & 0.12 & 0.12 \\
\hline
\end{tabular}

\begin{tabular}{|c|c|c|c|c|}
\hline Mean absolute value $\left[\mathrm{m} / \mathrm{s}^{2}\right]$ & 0.01 & 0.01 & 0.04 & 0.02 \\
\hline Maximum amplitude $\left[\mathrm{m} / \mathrm{s}^{2}\right]$ & 0.03 & 0.03 & 0.26 & 0.05 \\
\hline Median frequency $[\mathrm{Hz}]$ & 10.42 & 10.10 & 10.87 & 10.20 \\
\hline Mean frequency [Hz] & 13.74 & 11.32 & 13.69 & 17.13 \\
\hline Peak frequency [Hz] & 0.00 & 0.00 & 0.49 & 0.13 \\
\hline Electrode & 5 & 6 & 7 & 8 \\
\hline No. of zero crossings & 138 & 126 & 130 & 118 \\
\hline Mean waveform length $[\mathrm{s}]$ & 0.13 & 0.14 & 0.14 & 0.15 \\
\hline Mean absolute value $\left[\mathrm{m} / \mathrm{s}^{2}\right]$ & 0.01 & 0.00 & 0.00 & 0.00 \\
\hline Maximum amplitude $\left[\mathrm{m} / \mathrm{s}^{2}\right]$ & 0.04 & 0.02 & 0.01 & 0.01 \\
\hline Median frequency [Hz] & 9.80 & 9.80 & 10.64 & 9.80 \\
\hline Mean frequency [Hz] & 16.42 & 19.01 & 17.79 & 13.38 \\
\hline Peak frequency [Hz] & 0.00 & 0.00 & 0.00 & 0.00 \\
\hline
\end{tabular}

It can be noticed that in the case of the test all received signal features have low values, which shows also low activity of forearm muscles performing the test scenario. The last tests in a series showed that further iterations include fatigue which since the $10^{\text {th }}$ examination is noticeable.

\subsection{Evaluation Test Scenario \#2}

During the test, the tester put his hand into slight, regular tremors. Compared to the previous test case, it is possible to observe the increase in the mean absolute value and the maximum amplitude for the accelerometer. It can also be noticed that hand-shaking was accomplished primarily with the forearm muscle located under the $3^{\text {rd }}$ electrode. For that specific channel, sEMG values are significantly higher.

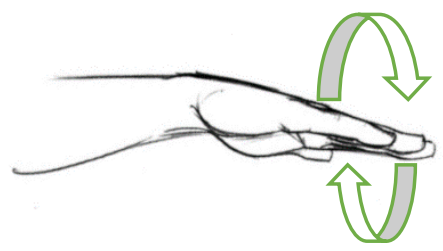

Fig. 8. Test gesture \#2 - A gentle hand shake resembling tremor

Table 2. ACC-sEMG signal features gathered for test gesture \#2 (avg values).

\begin{tabular}{|c|c|c|c|c|}
\hline \multicolumn{5}{|c|}{ Accelerometer } \\
\hline \multicolumn{2}{|l|}{ Axis } & $\mathbf{X}$ & $\mathbf{Y}$ & $\mathbf{Z}$ \\
\hline \multicolumn{2}{|l|}{ No. of zero crossings } & 182 & 126 & 189 \\
\hline \multicolumn{2}{|l|}{ Mean waveform length [s] } & 0.10 & 0.15 & 0.10 \\
\hline \multicolumn{2}{|l|}{ Mean absolute value $\left[\mathrm{m} / \mathrm{s}^{2}\right]$} & 0.32 & 1.30 & 0.29 \\
\hline \multicolumn{2}{|l|}{ Maximum amplitude $\left[\mathrm{m} / \mathrm{s}^{2}\right]$} & 1.01 & 4.22 & 1.02 \\
\hline \multicolumn{2}{|l|}{ Median frequency [Hz] } & 8.85 & 6.29 & 12.20 \\
\hline \multicolumn{2}{|l|}{ Mean frequency [Hz] } & 14.53 & 7.79 & 14.92 \\
\hline \multicolumn{2}{|l|}{ Peak frequency [Hz] } & 0.20 & 1.19 & 0.41 \\
\hline \multicolumn{5}{|c|}{ Electromyograph } \\
\hline Electrode & 1 & 2 & 3 & 4 \\
\hline No. of zero crossings & 187 & 178 & 185 & 189 \\
\hline Mean waveform length [s] & 0.10 & 0.10 & 0.10 & 0.10 \\
\hline Mean absolute value $\left[\mathrm{m} / \mathrm{s}^{2}\right]$ & 0.01 & 0.03 & 0.05 & 0.02 \\
\hline Maximum amplitude $\left[\mathrm{m} / \mathrm{s}^{2}\right]$ & 0.07 & 0.18 & 0.44 & 0.14 \\
\hline Median frequency [Hz] & 10.64 & 11.11 & 13.16 & 15.62 \\
\hline Mean frequency $[\mathrm{Hz}]$ & 19.91 & 17.04 & 17.72 & 19.09 \\
\hline Peak frequency [Hz] & 0.00 & 0.53 & 0.61 & 0.28 \\
\hline Electrode & 5 & 6 & 7 & 8 \\
\hline No. of zero crossings & 178 & 167 & 157 & 175 \\
\hline
\end{tabular}




\begin{tabular}{|l|l|l|l|l|}
\hline Mean waveform length $[\mathbf{s}]$ & 0.10 & 0.11 & 0.12 & 0.11 \\
\hline Mean absolute value $\left[\mathbf{m} / \mathbf{s}^{2}\right]$ & 0.01 & 0.01 & 0.01 & 0.01 \\
\hline Maximum amplitude $\left[\mathbf{m} / \mathbf{s}^{\mathbf{2}}\right]$ & 0.10 & 0.04 & 0.06 & 0.02 \\
\hline Median frequency $[\mathbf{H z}]$ & 12.05 & 10.42 & 10.42 & 10.53 \\
\hline Mean frequency $[\mathbf{H z}]$ & 15.13 & 13.16 & 14.80 & 16.09 \\
\hline Peak frequency $[\mathbf{H z}]$ & 5.23 & 0.00 & 0.00 & 0.00 \\
\hline
\end{tabular}

\subsection{Evaluation Test Scenario \#3}

The test scenario assumes clenching fist (strong forearm muscles activation) with all the strength, which imitates seizure tonic phase.

Based on the signal from the accelerometer it can be observed that during the tests, a slight hand tremor had been registered. A significant change in $\mathrm{SEMG}$ signal was recorded. Signal features for all sensor channels show higher values compared to previous test cases. For each sEMG channel, values are similar, which shows that testers were able to activate full set of forearm muscles.

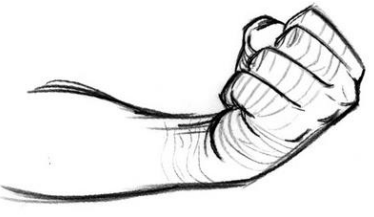

Fig. 9. Test gesture \#3 - Tightly clenched fist (tonic phase imitation)

Table 3. ACC-sEMG signal features gathered for test gesture \#3 (avg values).

\begin{tabular}{|c|c|c|c|c|}
\hline \multicolumn{5}{|c|}{ Accelerometer } \\
\hline \multicolumn{2}{|l|}{ Axis } & $\mathbf{X}$ & $\mathbf{Y}$ & $\mathbf{Z}$ \\
\hline \multicolumn{2}{|l|}{ No. of zero crossings } & 394 & 318 & 326 \\
\hline \multicolumn{2}{|l|}{ Mean waveform length [s] } & 0.04 & 0.06 & 0.06 \\
\hline \multicolumn{2}{|l|}{ Mean absolute value $\left[\mathrm{m} / \mathrm{s}^{2}\right]$} & 0.95 & 0.25 & 0.17 \\
\hline \multicolumn{2}{|l|}{ Maximum amplitude $\left[\mathrm{m} / \mathrm{s}^{2}\right]$} & 4.37 & 1.07 & 0.87 \\
\hline \multicolumn{2}{|l|}{ Median frequency $[\mathrm{Hz}]$} & 25.00 & 24.39 & 24.39 \\
\hline \multicolumn{2}{|l|}{ Mean frequency [Hz] } & 23.65 & 21.50 & 21.94 \\
\hline \multicolumn{2}{|l|}{ Peak frequency [Hz] } & 0.47 & 0.89 & 0.33 \\
\hline \multicolumn{5}{|c|}{ Electromyograph } \\
\hline Electrode & 1 & 2 & 3 & 4 \\
\hline No. of zero crossings & 151 & 147 & 141 & 142 \\
\hline Mean waveform length [s] & 0.12 & 0.12 & 0.12 & 0.12 \\
\hline Mean absolute value $\left[\mathrm{m} / \mathrm{s}^{2}\right]$ & 0.19 & 0.14 & 0.06 & 0.14 \\
\hline Maximum amplitude $\left[\mathrm{m} / \mathrm{s}^{2}\right]$ & 0.45 & 0.45 & 0.41 & 0.45 \\
\hline Median frequency [Hz] & 10.10 & 10.42 & 10.31 & 10.10 \\
\hline Mean frequency [Hz] & 27.23 & 18.81 & 14.31 & 17.41 \\
\hline Peak frequency $[\mathrm{Hz}]$ & 0.60 & 0.54 & 0.59 & 0.53 \\
\hline Electrode & 5 & 6 & 7 & 8 \\
\hline No. of zero crossings & 138 & 146 & 147 & 155 \\
\hline Mean waveform length [s] & 0.13 & 0.12 & 0.12 & 0.11 \\
\hline Mean absolute value $\left[\mathrm{m} / \mathrm{s}^{2}\right]$ & 0.20 & 0.15 & 0.14 & 0.18 \\
\hline Maximum amplitude $\left[\mathrm{m} / \mathrm{s}^{2}\right]$ & 0.45 & 0.45 & 0.45 & 0.45 \\
\hline Median frequency [Hz] & 9.71 & 10.64 & 11.36 & 11.49 \\
\hline Mean frequency [Hz] & 16.43 & 19.37 & 14.90 & 20.46 \\
\hline Peak frequency $[\mathrm{Hz}]$ & 0.36 & 0.63 & 0.75 & 0.62 \\
\hline
\end{tabular}

\subsection{Evaluation Test Scenario \#4}

Test scenario \#4 consisted of vertical movements of open hand sustaining steady fingers.

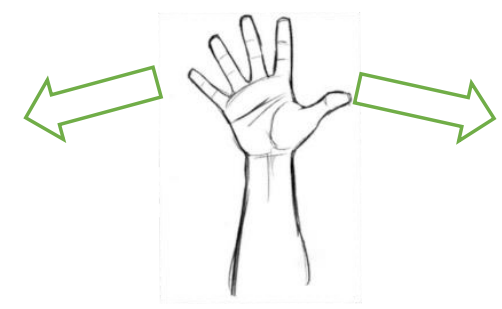

Fig. 10. Test gesture $\# 4-$ Waving an open hand

Table 4. ACC-sEMG signal features gathered for test gesture \#4 (avg values)

\begin{tabular}{|c|c|c|c|c|}
\hline \multicolumn{5}{|c|}{ Accelerometer } \\
\hline \multicolumn{2}{|l|}{ Axis } & $\mathbf{X}$ & $\mathbf{Y}$ & $\mathbf{Z}$ \\
\hline \multicolumn{2}{|l|}{ No. of zero crossings } & 145 & 67 & 133 \\
\hline \multicolumn{2}{|l|}{ Mean waveform length [s] } & 0.13 & 0.28 & 0.14 \\
\hline \multicolumn{2}{|l|}{ Mean absolute value $\left[\mathrm{m} / \mathrm{s}^{2}\right]$} & 0.33 & 1.15 & 0.47 \\
\hline \multicolumn{2}{|l|}{ Maximum amplitude $\left[\mathrm{m} / \mathrm{s}^{2}\right]$} & 1.36 & 3.00 & 1.52 \\
\hline \multicolumn{2}{|l|}{ Median frequency [Hz] } & 8.40 & 3.57 & 6.41 \\
\hline \multicolumn{2}{|l|}{ Mean frequency [Hz] } & 11.96 & 3.63 & 7.68 \\
\hline \multicolumn{2}{|l|}{ Peak frequency [Hz] } & 0.40 & 1.26 & 1.61 \\
\hline \multicolumn{5}{|c|}{ Electromyograph } \\
\hline Electrode & 1 & 2 & 3 & 4 \\
\hline No. of zero crossings & 183 & 187 & 177 & 174 \\
\hline Mean waveform length [s] & 0.10 & 0.10 & 0.10 & 0.11 \\
\hline Mean absolute value $\left[\mathrm{m} / \mathrm{s}^{2}\right]$ & 0.01 & 0.02 & 0.11 & 0.03 \\
\hline Maximum amplitude $\left[\mathrm{m} / \mathrm{s}^{2}\right]$ & 0.07 & 0.13 & 0.44 & 0.26 \\
\hline Median frequency [Hz] & 15.15 & 15.62 & 10.42 & 12.50 \\
\hline Mean frequency $[\mathrm{Hz}]$ & 14.08 & 17.08 & 19.21 & 16.28 \\
\hline Peak frequency $[\mathrm{Hz}]$ & 0.00 & 0.58 & 0.79 & 0.63 \\
\hline Electrode & 5 & 6 & 7 & 8 \\
\hline No. of zero crossings & 178 & 184 & 164 & 179 \\
\hline Mean waveform length [s] & 0.10 & 0.10 & 0.11 & 0.10 \\
\hline Mean absolute value $\left[\mathrm{m} / \mathrm{s}^{2}\right]$ & 0.02 & 0.01 & 0.01 & 0.01 \\
\hline Maximum amplitude $\left[\mathrm{m} / \mathrm{s}^{2}\right]$ & 0.09 & 0.11 & 0.06 & 0.09 \\
\hline Median frequency [Hz] & 12.50 & 10.53 & 10.53 & 13.51 \\
\hline Mean frequency $[\mathrm{Hz}]$ & 15.43 & 17.54 & 15.35 & 17.84 \\
\hline Peak frequency [Hz] & 0.28 & 0.00 & 0.00 & 0.80 \\
\hline
\end{tabular}

Based on the signal from the accelerometer, it is clearly visible that the hand movement was carried out primarily in one axis. The curves calculated for the $\mathrm{Y}$ axis are much larger than for other axes. This is consistent with expectations as the researcher moved his hand from side to side in a straight line. The analysis of the electromyograph signal shows that the hand waving was carried out mainly by the muscles located under the electrode number 3. This is similar as the conclusion determined in the case of Test 2 , in which shaking of the hand was a result of similar muscles.

\subsection{Evaluation Test Scenario \#5}

The next test consisted of bending the open palm to the back. The hand was held still.

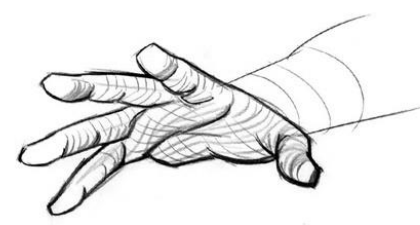

Fig. 11. Test gesture \#5 - Deflection of hand to back 
Table 5. ACC-sEMG signal features gathered for test gesture \#5 (avg values)

\begin{tabular}{|c|c|c|c|c|}
\hline \multicolumn{5}{|c|}{ Accelerometer } \\
\hline \multicolumn{2}{|l|}{ Axis } & $\mathbf{X}$ & $\mathbf{Y}$ & $\mathbf{Z}$ \\
\hline \multicolumn{2}{|l|}{ No. of zero crossings } & 275 & 274 & 225 \\
\hline \multicolumn{2}{|l|}{ Mean waveform length [s] } & 0.07 & 0.07 & 0.08 \\
\hline \multicolumn{2}{|l|}{ Mean absolute value $\left[\mathrm{m} / \mathrm{s}^{2}\right]$} & 0.09 & 0.07 & 0.04 \\
\hline \multicolumn{2}{|l|}{ Maximum amplitude $\left[\mathrm{m} / \mathrm{s}^{2}\right]$} & 0.85 & 0.42 & 0.22 \\
\hline \multicolumn{2}{|l|}{ Median frequency [Hz] } & 23.26 & 23.26 & 12.99 \\
\hline \multicolumn{2}{|l|}{ Mean frequency [Hz] } & 24.43 & 21.19 & 22.91 \\
\hline \multicolumn{2}{|l|}{ Peak frequency [Hz] } & 2.51 & 0.00 & 0.00 \\
\hline \multicolumn{5}{|c|}{ Electromyograph } \\
\hline Electrode & 1 & 2 & 3 & 4 \\
\hline No. of zero crossings & 172 & 172 & 179 & 161 \\
\hline Mean waveform length [s] & 0.11 & 0.11 & 0.10 & 0.12 \\
\hline Mean absolute value $\left[\mathrm{m} / \mathrm{s}^{2}\right]$ & 0.02 & 0.08 & 0.16 & 0.15 \\
\hline Maximum amplitude $\left[\mathrm{m} / \mathrm{s}^{2}\right]$ & 0.09 & 0.40 & 0.45 & 0.45 \\
\hline Median frequency [Hz] & 13.33 & 12.20 & 11.76 & 12.66 \\
\hline Mean frequency [Hz] & 19.86 & 21.23 & 26.25 & 16.09 \\
\hline Peak frequency $[\mathrm{Hz}]$ & 1.94 & 0.59 & 0.44 & 0.58 \\
\hline Electrode & 5 & 6 & 7 & 8 \\
\hline No. of zero crossings & 171 & 168 & 162 & 162 \\
\hline Mean waveform length [s] & 0.11 & 0.11 & 0.12 & 0.12 \\
\hline Mean absolute value $\left[\mathrm{m} / \mathrm{s}^{2}\right]$ & 0.07 & 0.02 & 0.01 & 0.01 \\
\hline Maximum amplitude $\left[\mathrm{m} / \mathrm{s}^{2}\right]$ & 0.31 & 0.11 & 0.08 & 0.06 \\
\hline Median frequency [Hz] & 12.05 & 11.76 & 11.76 & 11.49 \\
\hline Mean frequency [Hz] & 22.07 & 21.20 & 18.53 & 17.28 \\
\hline Peak frequency [Hz] & 0.65 & 0.80 & 0.00 & 0.00 \\
\hline
\end{tabular}

Based on the maximum amplitude, it is possible to observe high intensity tremors. The accelerometer signal correlated with sEMG demonstrates that the bending of the hand to the back engages muscles located under the electrodes 2, 3, 4. Obtained values from these channels vary compared to the other electrodes.

\subsection{Evaluation Test Scenario \#6}

The tester held his fist clenched and kept strained muscles while simultaneously making circles with the hand.

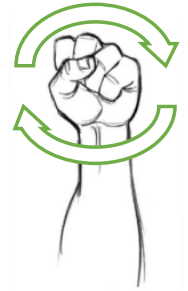

Fig. 12. Test gesture $\# 6$ - Waving a clenched fist

Table 6. ACC-sEMG signal features gathered for test gesture \#6 (avg values)

\begin{tabular}{|c|c|c|c|}
\hline \multicolumn{4}{|c|}{ Accelerometer } \\
\hline Axis & $\mathbf{X}$ & $\mathbf{Y}$ & $\mathbf{Z}$ \\
\hline No. of zero crossings & 131 & 96 & 96 \\
\hline Mean waveform length [s] & 0.14 & 0.19 & 0.20 \\
\hline Mean absolute value $\left[\mathrm{m} / \mathrm{s}^{2}\right]$ & 1.82 & 3.08 & 3.19 \\
\hline Maximum amplitude $\left[\mathrm{m} / \mathrm{s}^{2}\right]$ & 6.77 & 8.66 & 8.38 \\
\hline Median frequency [Hz] & 6.49 & 5.00 & 5.00 \\
\hline Mean frequency [Hz] & 9.83 & 5.61 & 5.37 \\
\hline Peak frequency [Hz] & 0.42 & 0.41 & 0.45 \\
\hline \multicolumn{4}{|c|}{ Electromyograph } \\
\hline Electrode & 2 & 3 & 4 \\
\hline
\end{tabular}

\begin{tabular}{|c|c|c|c|c|}
\hline No. of zero crossings & 150 & 173 & 187 & 167 \\
\hline Mean waveform length $[\mathbf{s}]$ & 0.12 & 0.11 & 0.10 & 0.11 \\
\hline Mean absolute value $\left[\mathrm{m} / \mathrm{s}^{2}\right]$ & 0.10 & 0.09 & 0.03 & 0.06 \\
\hline Maximum amplitude $\left[\mathrm{m} / \mathrm{s}^{2}\right]$ & 0.45 & 0.40 & 0.16 & 0.31 \\
\hline Median frequency $[\mathrm{Hz}]$ & 9.90 & 12.50 & 12.66 & 10.64 \\
\hline Mean frequency [Hz] & 25.98 & 21.68 & 17.71 & 22.75 \\
\hline Peak frequency [Hz] & 0.44 & 0.80 & 0.44 & 0.30 \\
\hline Electrode & 5 & 6 & 7 & 8 \\
\hline No. of zero crossings & 175 & 169 & 183 & 153 \\
\hline Mean waveform length [s] & 0.11 & 0.11 & 0.10 & 0.12 \\
\hline Mean absolute value $\left[\mathrm{m} / \mathrm{s}^{2}\right]$ & 0.04 & 0.05 & 0.05 & 0.07 \\
\hline Maximum amplitude $\left[\mathrm{m} / \mathrm{s}^{2}\right]$ & 0.19 & 0.45 & 0.45 & 0.41 \\
\hline Median frequency $[\mathrm{Hz}]$ & 12.66 & 10.75 & 12.99 & 10.20 \\
\hline Mean frequency [Hz] & 28.89 & 21.25 & 20.29 & 14.35 \\
\hline Peak frequency $[\mathrm{Hz}]$ & 0.40 & 0.57 & 0.45 & 0.53 \\
\hline
\end{tabular}

The accelerometer data prove, that the hand was moved mainly around two axes. This was due to the fact that the hand was rotating in the air. The signal from the electromyograph shows that the muscles were stressed during the test, because the values for each channel are close to those obtained during the Test \#3.

\subsection{Summary of obtained test results}

Conducted tests have proven the adequacy of sEMG and inertial sensor monitoring for tremor, bradykinesia and dyskinesia recording and evaluation. Wearable sensor provide very useful tool for supplementing surveyoriented approach for evaluating health state of PD patients, and the intensity of disease symptoms. It should be also stated that some part of PD patients may not suffer from these type of symptoms, thus the method requires screening the clinical trials participants. Utilisation of the inertial and electromyography signals can be an efficient way of gestures and disease identification. Provided in the paper extension of previous approach allowed the authors to increase significantly the method's accuracy and enrich pattern descriptions. The approach provided not only the force of movement, but also direction in which the hand (forearm) moved (test scenarios 2,4,6). Supplementing inertial data with sEMG has proven to augment gesture interpretation. More accurate hand movements detection can be utilised for tremor, bradykinesia and rigidness detection after supplementing the calibration data for a given patient defining the $\mathrm{ON}$ and $\mathrm{OFF}$ state characteristics - examples (tests scenarios 2,3,5). Further analysis of the obtained experiences shows that application of forms of machine learning techniques can support more accurate movement recognition thus helping with more accurate, sophisticated disease symptoms recognition. More accurate algorithms provide also means for evaluating the intensity of disease symptoms ( $\mathrm{PG}$, epilepsy), which can be also used for therapy inertia assessment.

\section{Conclusions and further research}

Provided methodology, research results and tool itself deliver means for clinical trials data acquisition and assessment. Provided 3 level evaluation (survey, sensor, interactions) supplement each other and deliver wide spectrum of tools for assisting therapy. Elaborated 
analytical method and obtained conclusions demonstrate the applicability of mobile devices with closely coupled dedicated software, guiding the patient through efficient health state reporting. Based on analogue clinical trials observations, a set of requirements for validating symptoms of neurological diseases have been formulated, concentrating on the ones which can be registered using wearable sensors. PATRON delivers extended, configurable scheduling options, for trials managers to adjust specific test parameters. A calibration of algorithms supports patient personalisation, which enables more accurate recognition of significant health states but also supports symptom's intensity evaluation. Developed mobile system is aimed at clinical trials assistance, while constantly aggregating gathered surveying and sensor data. Such quantitative approach is a supplement for patient's subjective evaluation of health state, which makes this method attractive for pharmaceutical market. This work provided also discussion on pros and cons of CT process composition and its supplementation with mobile and wearable technology - which in the end delivers innovative and trustworthy research utilities. Paper provides summary of analytical recommendations associated with the effectiveness and usefulness of selected sensor signals and monitored symptoms. Presented research introduces also new ideas and techniques to supplement examinations with tailored exercises as well as reflex evaluations. Further research will be aimed at development of multi-criteria decision procedures for symptoms recognition and intensity evaluation deployed on mobile platforms.

Acknowledgements: This work was supported by supported by the EU grant POIR.02.03.02-14-0055/17-00 Development of an innovative IT solution to support the process of clinical trials associated with Parkinson's disease.

\section{References}

1. M. Chmielewski, M. Nowotarski, Sensor-based supporting mobile system Parkinson disease clinical tests utilising biomedical and RFID technologies, MATEC Web Conf., vol. 125, (2017)

2. R. LeMoyne, Wearable and wireless accelerometer systems for monitoring Parkinson's disease patients A perspective review, Advances in Parkinson's Disease, 2, 113-115 (2013)

3. S.H. Roy, B.T. Cole, L.D. Gilmore, C.J. De Luca, C.A. Thomas, M.M. Saint-Hilaire, S.H. Nawab: High-Resolution Tracking of Motor Disorders in Parkinson's Disease During Unconstrained Activity, Movement Disorders, 28, 1080-1087 (2013)

4. S. Beniczky, T. Polster, T. W. Kjaer, H. Hjalgrim: Detection of generalized tonic-clonic seizures by a wireless wrist accelerometer: A prospective, multicenter study, Epilepsia, 54, 58-61 (2013)

5. M.M. Velez, R.S. Fisher, V. Bartlett, S. Le: Tracking generalized tonic-clonic seizures with a wrist accelerometer linked to an online database, Seizure, 39, 13-18 (2016)
6. J.R. Villar, M. Menendez, J. Sedano, E. de la Cal, V.M. Gonzalez: Analyzing accelerometer data for epilepsy episode recognition, 10th International Conference on Soft Computing Models in Industrial and Environmental Applications. Advances in Intelligent Systems and Computing, 368, 39-48, Springer, Cham (2015)

7. SENSE project Wiki: http://uranus.wat.edu.pl:8808/wiki/index.php/ SENSE (access: 2018.05.05)

8. PATRON project wiki: http://uranus.wat.edu.pl:8808/wiki/index.php/ PATRON (access: 2018.05.05)

9. Myo tech spec: (access: 2018.05.05) https://www.myo.com/techspecs

10. B. Stern: Inside Myo, adafruit.com (2016): https://learn.adafruit.com/myo-armbandteardown/inside-myo

11. Myo Bluetooth Low Energy specification file: https:/github.com/thalmiclabs/myobluetooth/blob/master/myohw.h

12. M. Abduo, M. Galster: Myo Gesture Control Armband for Medical Applications, University of Canterbury (2015)

13. A. Phinyomark, P. Phukpattaranont, C. Limsakul, Feature reduction and selection for EMG signal classification, Expert Systems with Applications, vol. 39, (8), 2012, pp. 7420-7431

14. M.B.I. Raez, M.S. Hussain, F. Mohd-Yasin, Techniques of EMG signal analysis: detection, processing, classification and applications, Biol Proced Online, 2006, vol. 8, pp. 11-35.

15. M. Kiedrowicz, Location with the use of the RFID and GPS technologies - opportunities and threats, GIS ODYSSEY 2016, pp. 122-128, (2016).

16. M. Kiedrowicz, Objects identification in the informations models used by information systems, GIS ODYSSEY 2016, pp. 129-136, (2016).

17. M. Chmielewski, M. Nowotarski, The method for processing electromyography and inertial sensors supporting chosen set of neurological symptoms for clinical trials support and treatment assessment, WSEAS TRANSACTIONS on BIOLOGY and BIOMEDICINE, vol. 14, (2017)

18. M. Nowotarski: Development of decision algorithms using selected inertial and biomedical signals to identify neurological disorders utilising Android mobile platform., master's thesis, M. Chmielewski (supervisor), MUT, 2016, Warsaw

19. R. Boostani M. H. Moradi, Evaluation of the forearm EMG signal features for the control of a prosthetic hand, Physiol Meas., 2003, 24(2), pp.309-319

20. J. R. Wilkinson, handbook of Parkinson's Disease, 4th edition, Neurology, 2008, 71 (9) 699; 